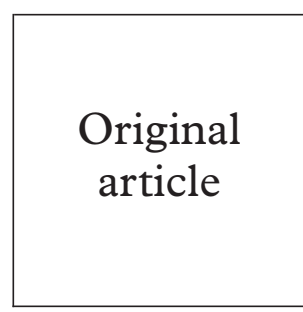

Institute for Research in Extramural

Medicine, Vrije

Universiteit,

Amsterdam,

Netherlands

I $G M$ van Valkengoed

L M Bouter

A J P Boeke

Groningen University Institute for Drug

Exploration,

Groningen,

Netherlands

M J Postma

University Hospital

Vrije Universiteit,

Department of

Pathology, Section of

Molecular Pathology,

Amsterdam,

Netherlands

S A Morré

A J C van den Brule

C J L M Meijer

Correspondence to:

Dr A J P Boeke, Institute for Research in Extramural

Medicine, Vrije Universiteit,

Van der Boechorststraat 7,

1081 BT Amsterdam,

Netherlands

ajp.boeke.emgo@med.vu.nl

Accepted for publication

3 April 2001

\title{
Cost effectiveness analysis of a population based screening programme for asymptomatic Chlamydia trachomatis infections in women by means of home obtained urine specimens
}

\author{
Irene G M van Valkengoed, Maarten J Postma, Servaas A Morré, Adriaan J C van den \\ Brule, Chris J L M Meijer, Lex M Bouter, A Joan P Boeke
}

Objectives: To evaluate the cost effectiveness of a systematic screening programme for asymptomatic Chlamydia trachomatis infections in a female inner city population. To determine the sensitivity of the cost effectiveness analysis to variation in the probability of developing sequelae.

Methods: A decision tree was constructed to evaluate health effects of the programme, such as averted sequelae of chlamydial infection. Cost effectiveness from a societal perspective was estimated for screening by means of a ligase chain reaction on mailed, home obtained urine specimens, in a population with a $C$ trachomatis test prevalence of $2.9 \%$. An extensive sensitivity analysis was performed for the probability of sequelae, the percentage of preventable pelvic inflammatory disease (PID), and the discount rate.

Results: The estimated net cost of curing one woman, aged 15-40 years, of a $C$ trachomatis infection is US $\$ 1210$. To prevent one major outcome (PID, tubal factor infertility, ectopic pregnancy, chronic pelvic pain, or neonatal pneumonia), 479 women would have to be screened. The net cost of preventing one major outcome is $\$ 15800$. Changing the probability of PID after chlamydial infection from $5 \%$ to $25 \%$ decreases the net cost per major outcome averted from $\$ 28300$ to $\$ 6380$, a reduction of $78 \%$. Results were less sensitive to variations in estimates for other sequelae. The breakeven prevalence of the programme ranges from $6.4 \%$ for the scenario with all probabilities for complications set at the maximum value to a prevalence of $100 \%$ for probabilities set at the minimum value.

Conclusions: Systematic screening of all women aged 15-40 years for asymptomatic $C$ trachomatis infections is not cost effective. Although the results of the analyses are sensitive to variation in the assumptions, the costs exceed the benefits, even in the most optimistic scenario.

(Sex Transm Inf 2001;77:276-282)

Keywords: cost effectiveness analysis; Chlamydia trachomatis; screening

\section{Introduction}

Genital infections caused by the bacteria Chlamydia trachomatis are the most common sexually transmitted infections in industrialised countries. In women, $C$ trachomatis can cause urethritis, cervicitis, pelvic inflammatory disease (PID) and, at a later stage, tubal factor infertility (TFI), ectopic pregnancy (EP), and chronic pelvic pain (CPP). ${ }^{12}$ When giving birth an infected woman can pass the infection on to her child, who can develop a neonatal conjunctivitis or pneumonia as a result of the infection. $^{3}$

Since genital chlamydial infection causes no or few symptoms, many infections remain undetected. Delayed treatment increases the risk of sequelae and transmission to sex partners. Screening is therefore indicated to prevent the spread of $C$ trachomatis and also to prevent complications. Research in the United States and Sweden has shown that the detection and treatment of asymptomatic infections in women results in a reduction of complications. ${ }^{45}$

Cost is an important factor, which should be taken into consideration before a screening programme is implemented. Cost effectiveness analyses of opportunistic screening programmes, focusing on patients attending healthcare clinics, or specific high risk groups, have shown that screening becomes cost effective at prevalences ranging from $2 \%$ to $6 \%{ }^{6-8}$ The introduction of sensitive DNA detection methods to test non-invasive specimens, such as urine, and the testing of mailed home obtained samples makes it possible to extend screening beyond the traditional settings. ${ }^{9-12}$ The cost effectiveness of systematic screening programmes in the general population has not been evaluated.

In most cost effectiveness studies to date the costs of organising the screening, including administrative costs, have not been included in the analysis. Furthermore, although some studies mention the imperfect specificity of the test, no study has taken the cost of follow up of false positive test results into account in the analysis. So far, only one study has reported a sensitivity analysis of risk estimates, although estimating the probability of complications based on the available literature leaves much room for variation. ${ }^{7}$

This paper reports on the cost effectiveness of a systematic screening programme for asymptomatic $C$ trachomatis infections in 


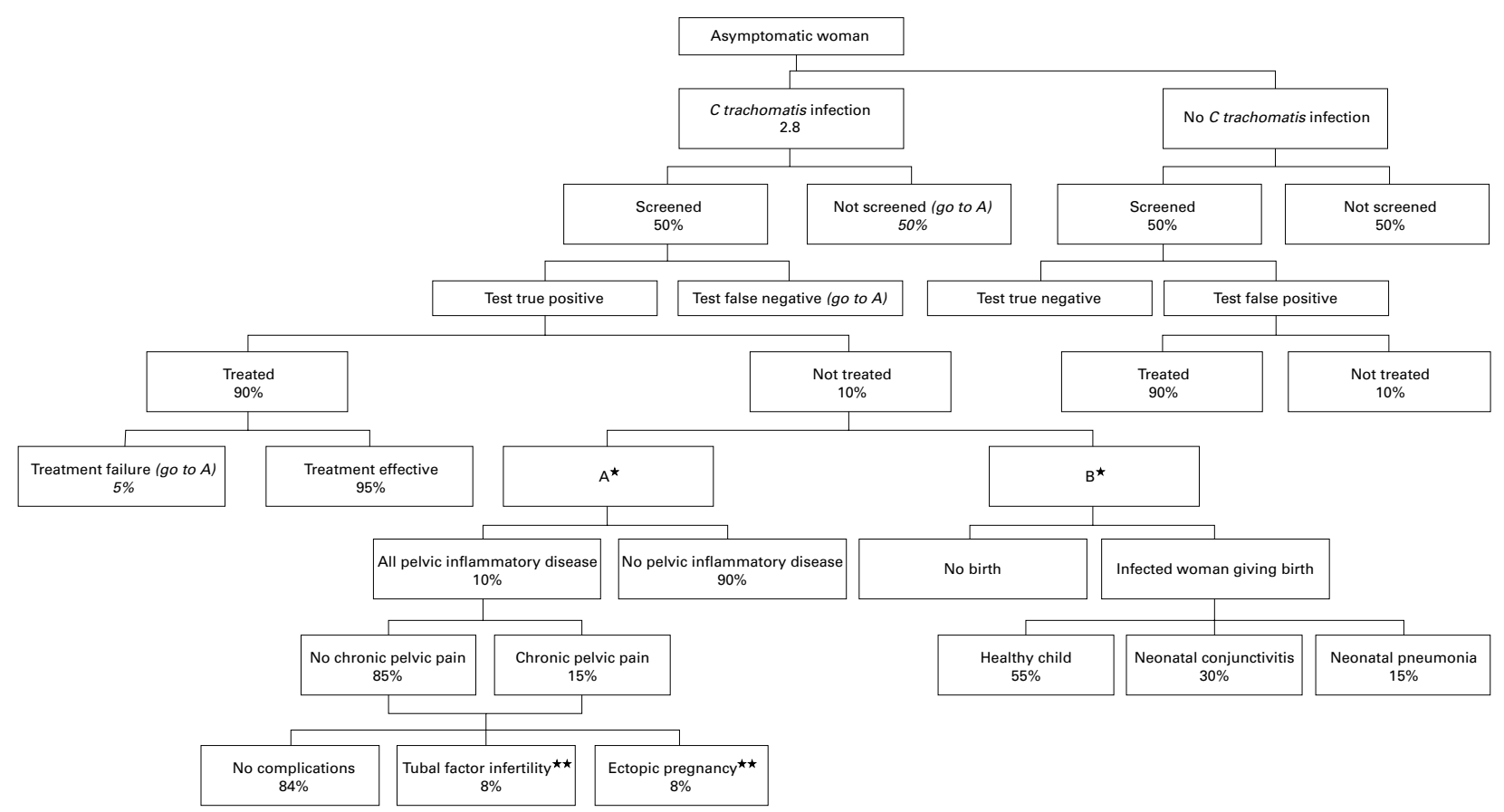

Figure 1 Decision model for a population based screening programme for asymptomatic Chlamydia trachomatis infections in women. ${ }^{\star}$ Both $A$ and $B$ apply for all infected women. ${ }^{\star *}$ Pregnancy wish is taken into account.

women registered in general practice, based on mailed home obtained urine specimens. The sensitivity of the cost effectiveness analysis to variation in the probability of developing PID, EP, TFI, and CPP was determined.

\section{Methods}

RECRUITMENT

A random sample of 5541 women, aged 15-40 years, was selected from the computer registers of 16 inner city general practices in Amsterdam. Between November 1996 and October 1997 these women were invited by their general practitioner to participate in the screening programme. Coded research material was sent to the home address of potential participants. Participants were requested to mail a first void, first stream urine sample and a completed questionnaire to the department of pathology in a prestamped addressed envelope, which was included in the material.

The study has been approved by the medical ethics committee of the Vrije Universiteit in Amsterdam.

DIAGNOSIS OF INFECTION

Urine samples were tested for the presence of $C$ trachomatis by means of the ligase chain reaction (LCR) (Abbott Laboratories, Chicago, IL, USA) in the laboratory of the department of pathology of the University Hospital Vrije Universiteit. ${ }^{9-12}$ Tests were performed according to the instructions of the manufacturer.

The test results were reported back to the general practitioners, who were instructed to treat infected patients with a single dose of $1000 \mathrm{mg}$ azithromycin, or erythromycin for pregnant women $(4 \times 500 \mathrm{mg}$ for 5 days $)$.
CHARACTERISTICS OF PARTICIPANTS

Fifty per cent of the women who were invited participated in the screening programme; $25 \%$ of the participants were of non-Dutch origin. Of all participating women, 120 had never been sexually active. The prevalence of asymptomatic infection, as determined by means of the LCR on urine for women aged 15-20 years, 21-25 years, 26-30 years, 31-35 years, and $36-40$ years was $2.4 \%, 4.4 \%, 2.8 \%, 2.5 \%$, and $2.2 \%$, respectively.

\section{DECISION TREE ANALYSIS}

Figure 1 is a diagram of the decision tree used in the analysis. The sensitivity of the LCR on urine was estimated to be $85 \%$, and the specificity $99 \% .{ }^{9}{ }^{10}$ The assumptions for the risk of an untreated $C$ trachomatis are listed in table 1. The risk of developing sequelae if treatment fails is assumed to equal the risk if a woman has not been tested or treated effectively.

As shown in figure 1 and table 1 , the probability of pregnancy wish is age specific. ${ }^{15}$ This was included in the model to estimate how many women would develop an EP or consult a specialist for TFI. In addition, only women who gave birth while infected with $C$ trachomatis were included in calculations of the probability of having a child with neonatal conjunctivitis or neonatal pneumonia..$^{15}$ For the infectious period, an average of 52 weeks was assumed. ${ }^{16}$

\section{DIRECT COSTS}

The administrative costs and the costs of the materials sent to all women who were invited, were taken into account in the model (table 2).

Based on the workload reported by participating practices, it was estimated that the programme could be coordinated by two social nurses, both working full time. The recruitment costs for the programme have to be 
calculated for all women who were invited, since the material was not only sent to participants, but to all eligible women. The cost of recruitment is the sum of the cost of a urine container, an envelope, and postage (approximately US $\$ 0.50)$. For participants, a cost of $\$ 17.29$ is included for return postage, and test and laboratory costs. The costs of follow up of an infected woman include the cost of one visit to a general practitioner, pharmacotherapy costs, and the pharmacist's fees. Treatment costs are also included for women with a false positive test result, although treating these women will not prevent any sequelae.

The cost estimates for sequelae are based on research carried out by Postma et al. ${ }^{17}$ It was assumed that $75 \%$ of women with symptomatic PID are treated on an outpatient basis, ${ }^{18}$ involving two visits to a GP, medication, a test for $C$ trachomatis, and a test for Neisseria gonorrhoea. It is assumed that the remaining $25 \%$ are treated on an inpatient basis for 9.6 days 18 , involving a diagnostic laparoscopy and medication. ${ }^{19}$

With regard to infertility, $66 \%$ of the women were assumed to seek evaluation, $20 \%$ of whom are evaluated in an inpatient setting. ${ }^{20}$ In vitro fertilisation (an average of 2.11 attempts) was assumed to occur in $23 \%$ of the infertile women evaluated.

Chronic pelvic pain was assumed to be treated with analgesic drugs, and with pelvic ultrasonography and ambulatory laparoscopy for $75 \%$ of the patients. ${ }^{6}$ Furthermore, outpatient treatment was assumed to involve 10 appointments with a physician (three with a GP, seven with a gynaecologist). The assumption for inpatient care was two appointments with a gynaecologist and hospitalisation for the same duration as inpatient treatment of PID, preceded by one visit to a GP. Seventy per cent of cases of chronic pelvic pain were assumed to be treated on an outpatient basis, and $30 \%$ on an inpatient basis. ${ }^{6}$ Owing to lack of reliable information surgery was not taken into consideration.

Neonatal conjunctivitis was assumed to involve tests for $C$ trachomatis and $N$ gonorrhoea and medication. The assumption for outpatient care for neonatal pneumonia was one visit to a GP, two visits to a paediatrician, a test for $C$ trachomatis, and medication. One visit to a
GP and 13 days of intensive inpatient hospital care was assumed for $10 \%$ of cases of neonatal pneumonia. $^{6} 18$

Deflators for the gross domestic product (GDP) were used to recalculate all costs in 1996 prices. Costs were converted into US $\$$ on the basis of the GDP purchasing power parity (1 US $\$=2.07$ Dutch guilders). ${ }^{21}$ Estimated costs of a hospital inpatient day for intensive neonatal care are $\$ 1159$. $^{22}$ The costs of accommodation for a "normal" hospital inpatient day were estimated at $\$ 304 .^{23}$ Fees for examinations and treatment were used to cost hospital activities (data provided by the Academic Hospital in Groningen). The cost of one in vitro examination was estimated at $\$ 1423,{ }^{20}$ and the costing of medication was based on market prices for standard prescriptions. ${ }^{24}$ The weighted average GP charge for publicly and privately insured patients is $\$ 15.51 .^{25}$

\section{INDIRECT COSTS}

The indirect costs of $C$ trachomatis infections were estimated by applying the friction costs approach. To calculate a value for the loss of productivity from paid work, the average labour costs according to age and sex were used to approximate the net product. Based on an analysis of the labour costs in the Netherlands, the average gross income by age and sex were adjusted and inflated to derive the average labour costs applicable in the Netherlands in $1996 .{ }^{152}$ They were multiplied by the employed labour force participation rate, specified according to age and sex, which yielded the net product per capita from paid work. ${ }^{15}$ Based on data obtained from the literature, 10 days of productivity were estimated to be lost for each case of treatment for PID or chronic pelvic pain. Moreover, 21 days were estimated to be lost for each case of inpatient treatment for PID or chronic pelvic pain and 28 days for each case of ectopic pregnancy. ${ }^{7}$ Assuming that an ambulatory visit costs half a working day and an appointment with a GP 1.5 hours, the loss of productivity due to infertility management ( 5 days, 4.5 hours for outpatient management, 4.5 days for inpatient management), and in vitro fertilisation (4 days) was computed. Indirect costs due to the loss of unpaid work were not taken into consideration.

Table 1 Estimates and ranges used for the sensitivity analysis of the cost effectiveness of a universal screening programme for asymptomatic Chlamydia trachomatis infections in a female population

\begin{tabular}{lllll}
\hline Variable & Estimate (\%) & Range & Domain & References \\
\hline Prevalence & 2.9 & - & All women & Study data \\
Sensitivity LCR & 85 & $85-90$ & Tested women & 9,10 \\
Specificity LCR & 99 & - & Tested women & 8,10 \\
Contacted for treatment & 90 & - & Women with chlamydia & Study data \\
Successfully treated & 95 & - & Women with chlamydia, who receive & Study data \\
& 10 & $5-25$ & treatment & 4,13 \\
All PID & & & Women with undiagnosed or & 14 \\
& 50 & - & All PID & 14 \\
Asymptomatic of total PID & 50 & $25-75$ & All PID & 1 \\
Preventable PID & 8 & $4-16$ & All PID & 1 \\
Infertility & 8 & $4-16$ & All PID & 2 \\
Ectopic pregnancy & 15 & $10-20$ & All PID & 3 \\
Chronic pelvic pain & 30 & - & Women with chlamydia & 3 \\
Neonatal conjunctivitis & 15 & - & Women with chlamydia &
\end{tabular}

${ }^{\star}$ Discounted in the model by the age specific desire for pregnancy.

$\mathrm{LCR}=$ ligase chain reaction, $\mathrm{PID}=$ pelvic inflammatory disease. 
Table 2 Cost of the screening programme and direct and indirect costs associated with Chlamydia trachomatis infections and sequelae in women (price level 1996, in US\$)

\begin{tabular}{|c|c|c|}
\hline Variable & Direct (medical) cost & Indirect cost \\
\hline $\begin{array}{l}\text { Coordination of the program ( } 2 \text { fulltime social workers, } \\
\text { cost per person) }\end{array}$ & 48309 per year & - \\
\hline Recruitment cost* (container, envelope, postage) & 0.48 & - \\
\hline Laboratory test $\dagger$ (postage, materials, and labour) & 17.29 & - \\
\hline Treatment $(1000 \mathrm{mg}$ azithromycin) & 9.52 & - \\
\hline GP consultation & 15.51 & - \\
\hline \multicolumn{3}{|l|}{ Symptomatic PID } \\
\hline Inpatient treatment & 3908 & 276 \\
\hline Outpatient treatment & 67 & 132 \\
\hline \multicolumn{3}{|l|}{ Infertility $\ddagger$} \\
\hline Inpatient evaluation & 2315 & 212 \\
\hline Outpatient evaluation & 805 & 286 \\
\hline In vitro fertilisation $\ddagger$ & 1423 & 188 \\
\hline Ectopic pregnancy & 2909 & 847 \\
\hline \multicolumn{3}{|l|}{ Chronic pelvic pain $\ddagger$} \\
\hline Inpatient treatment & 3310 & 456 \\
\hline Outpatient treatment & 587 & 217 \\
\hline Neonatal conjunctivitis & 39 & - \\
\hline \multicolumn{3}{|l|}{ Neonatal pneumonia } \\
\hline Inpatient treatment & 16150 & - \\
\hline Outpatient treatment & 94 & 9 \\
\hline
\end{tabular}

^For every woman invited to participate.

†For every participant.

¥Costs presented in the table are for women aged 15-25 years and are not discounted. In the model, all future costs were discounted at a rate of $3 \%$ per year.

SUMMARY OF COSTS

Table 2 presents a summary of the cost estimates. In the model, all future costs were discounted at a rate of $3 \%$ per year. ${ }^{27}$ The cost of complications in newborn babies and the cost of PID were assigned to the year of infection. It was assumed that costs for chronic pelvic pain occur 5 years after PID. ${ }^{76}$ Costs for ectopic pregnancy and infertility are assumed to occur 10 years after $C$ trachomatis infection in women aged $15-19,5$ years after infection in women aged $20-24$, and 2 years after infection in older women.

Health benefits (averted complications) were not discounted, as has been suggested by Parsonage and Neuberger. ${ }^{28}$ It must be noted that discounting of health benefits is recommended by others. ${ }^{27}$

\section{SCREENING STRATEGIES}

The following screening strategies were taken into consideration: screening all women aged $15-25$, as recommended by the guidelines of the Centers for Disease Control, ${ }^{19}$ and screening all women aged 15-40. The cost effectiveness of a screening strategy was calculated as a weighted average of age specific results, weighted by the age distribution of women in Amsterdam.

The cost effectiveness is presented for two denominators: a woman cured and a major outcome averted (MOA). A cured woman is defined as an asymptomatically infected woman, detected by the screening programme, who was effectively treated for $C$ trachomatis. A major outcome is defined as either an episode of PID, CPP, EP, TFI, or neonatal pneumonia. Cost effectiveness is presented in a baseline analysis and in a sensitivity analysis.

\section{SENSITIVITY ANALYSIS}

Sensitivity analyses were performed for the probability of developing a PID after asymptomatic infection (range 5-25), the risk of developing TFI after PID (range 4-16), probability of developing chronic pelvic pain (range
10-20) and finally the probability of developing EP (range 4-16). The worst and best case scenario in terms of cost versus benefit were calculated by estimating the cost per major outcome averted with all the probabilities set at the minimum and maximum plausible values, respectively. Moreover, sensitivity analyses were applied to assumptions reflecting the preventable percentage of PIDs (from $75 \%$ to $25 \%$ ), test sensitivity (from $85 \%$ to $90 \%$ ), and discount rate (range $0-7 \%$ ). Sensitivity analysis was limited to the net cost per MOA in the screening programme aimed at 15-40 year old women.

No sensitivity analysis was performed for the cost of the test, since a discount for large scale screening was already included in the calculations of the costs of the test.

Breakeven prevalences were calculated for screening with baseline assumptions, minimum probabilities, and maximum probabilities. The breakeven prevalence reflects the prevalence where the costs of screening (US\$) equal those of averted healthcare costs.

\section{Results}

BASELINE ANALYSIS

As can be directly derived from the assumptions made, the probability of an infected woman being detected by the screening and being effectively treated is estimated at $73 \%$. The overall test prevalence of $2.9 \%$, found among women in Amsterdam corresponds with an estimated prevalence of $2.2 \%$, corrected for sensitivity and specificity of the test assumed in the model. In a screening programme aimed at women aged $15-40$ years, 889 women would have to be screened to prevent one major outcome. Given the current participation rate, approximately twice this number of women would have to be invited for screening. The implementation of screening for all women aged 15-40 years in Amsterdam $(n=161065)$ could prevent a total of 59 episodes of PID, three TFIs, six EPs, and nine cases of CPP, and could also prevent 13 cases 
Table 3 Cost effectiveness analysis of a screening programme for asymptomatic Chlamydia trachomatis infections in a low prevalence female population (in US\$)

\begin{tabular}{|c|c|c|c|c|}
\hline \multirow{2}{*}{$\begin{array}{l}\text { Age group } \\
\text { (years) }\end{array}$} & \multicolumn{2}{|c|}{ Net cost per woman cured } & \multicolumn{2}{|c|}{ Net cost per $M O A$} \\
\hline & Direct only & Total & Direct only & Total \\
\hline $15-25$ & 802 & 792 & 11200 & 11100 \\
\hline $15-40$ & 1220 & 1210 & 16000 & 15800 \\
\hline
\end{tabular}

MOA = major outcome averted (either an episode of PID chronic pelvic pain, ectopic pregnancy, tubal factor infertility, or neonatal pneumonia).

of neonatal pneumonia from occurring as a result of chlamydial infection.

The net cost per woman cured for a screening programme directed at women aged 15-40 years is $\$ 1210$ per woman cured (table 3 ).

Limiting the programme to women aged 15-25 years reduces the net cost per woman cured to $\$ 790$. Net costs per MOA are $\$ 15800$ and $\$ 11100$, respectively.

\section{SENSITIVITY ANALYSIS}

The results of the sensitivity analyses are presented in table 4 . Changing the estimate for the probability of developing PID after chlamydial infection from $5 \%$ to $25 \%$ decreases the cost per major outcome averted from $\$ 28300$ to $\$ 6380$, a reduction of $78 \%$. Similarly, plausible ranges for the probability of ectopic pregnancy, infertility, and chronic pelvic pain result in variations of $12 \%, 5 \%$, and $7 \%$, respectively. Changing the value of the percentage of PIDs that can be prevented by screening from $75 \%$ to $25 \%$ results in a $63 \%$ decrease in cost per major outcome averted. Assuming a further technical development of tests, a test sensitivity of $90 \%$ and a specificity of $99.9 \%$ were also taken into consideration. Increasing the assumed specificity resulted in a decrease in cost per major outcome averted from $\$ 15800$ to $\$ 9540$, a decrease of $40 \%$.

Finally, changing the discount rate from $0 \%$ to $7 \%$ results in an increase of $1 \%$ in cost per MOA.

BREAKEVEN PREVALENCE

Figure 2 shows the association between test prevalence and net cost per MOA of the programme aimed at women aged $15-40$ years. The breakeven prevalence in the baseline analysis was $41.8 \%$. The breakeven prevalences range from $6.4 \%$ for the best case scenario to $100 \%$ for the worst case scenario.

Table 4 Sensitivity analyses for a screening programme for women aged 15-40 years, variations in the probability estimates for sequelae, discount rate, and test characteristics (in US\$)

\begin{tabular}{lllll}
\hline Parameter & Range (\%) & $\begin{array}{l}\text { Cost per MOA } \\
(\text { min) }\end{array}$ & $\begin{array}{l}\text { Cost per MOA } \\
\text { (max) }\end{array}$ & $\begin{array}{l}\text { Change } \\
(\%)\end{array}$ \\
\hline Risk PID & $5-25$ & 28300 & 6380 & -78 \\
Risk EP & $4-16$ & 16500 & 14600 & -12 \\
Risk TFI & $4-16$ & 16100 & 15300 & -5 \\
Risk CPP & $10-20$ & 16400 & 15300 & -7 \\
Preventable PID & $25-75$ & 28300 & 10400 & -63 \\
Sensitivity of the test & $85-90$ & $15800^{\star}$ & 15800 & 0 \\
Specificity of the test & $99-99.9$ & $15800^{\star}$ & 9540 & -40 \\
Discount rate & $0-7$ & 15800 & 15900 & +1
\end{tabular}

$\mathrm{PID}=$ pelvic inflammatory disease, $\mathrm{EP}=$ ectopic pregnancy, $\mathrm{TFI}=$ tubal factor infertility, $\mathrm{CPP}=$ chronic pelvic pain, $\mathrm{MOA}=$ major outcome averted (either an episode of PID, CPP, EP, TFI, or neonatal pneumonia), $\min =$ minimum probabilities, $\max =$ maximum probabilities.

${ }^{\star}$ Estimate and resulting cost per MOA in the baseline analysis.

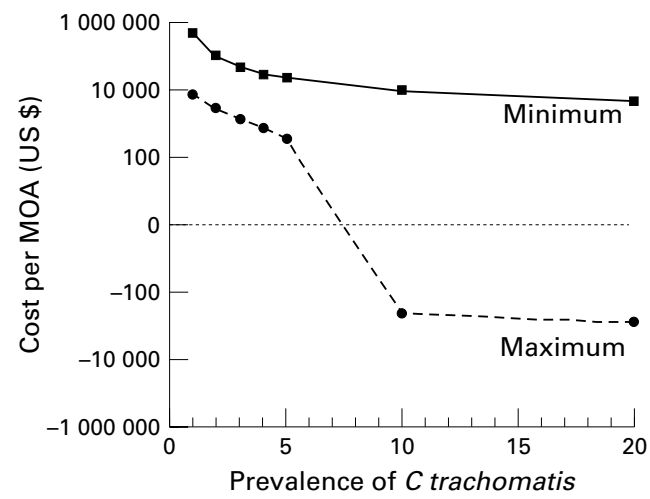

Figure 2 Breakeven prevalences for a screening programme for asymptomatic Chlamydia trachomatis infections in a female population: baseline assumptions, maximum probabilities, minimum probabilities. $M O A=$ major outcome averted (either an episode of pelvic inflammatory disease (PID), chronic pelvic pain, ectopic pregnancy, tubal factor infertility, or neonatal pneumonia). Maximum probabilities: assumption for all PID 25\%, tubal factor infertility 4\%, ectopic pregnancy 4\%, chronic pelvic pain 10\%. Minimum probabilities: assumption for all PID $5 \%$, tubal factor infertility $4 \%$, ectopic pregnancy $4 \%$, chronic pelvic pain $20 \%$.

The breakeven prevalence of a programme aimed at women aged 15-25 ranges from $6.1 \%$ to $100 \%$ in the sensitivity analysis.

As a test prevalence of $2.9 \%$ was found among 15-40 year old women in Amsterdam, the range of cost per MOA, reflecting the range in probabilities, would be $\$ 1514$ (maximum probabilities) to $\$ 48604$ (minimum probabilities). Similarly, at a test prevalence of $5 \%$, the interval for net cost per MOA would range from $\$ 338$ to $\$ 7485$.

\section{Discussion}

This is the first cost effectiveness analysis of a systematic, universal screening programme based on mailed home obtained urine specimens in the general population. The results show that the cost of a screening programme for women was found to exceed the benefits in both the baseline and the sensitivity analysis. Screening all women aged 15-40, under baseline assumptions, only becomes cost saving at a test prevalence of $41.8 \%$ or more.

The overall test prevalence in the study population was $2.9 \%$. Cost effectiveness for the screening programme directed at women aged $15-40$ years was estimated at $\$ 15800$ per MOA. Restriction of the programme to women aged 15-25 years lowers the net costs to \$11 100 per MOA. Another frequently used outcome in cost effectiveness analyses of chlamydia screening is cost per PID averted. PID constitutes $66.3 \%$ of the cost per MOA at baseline.

Selective screening is one way of increasing the efficiency of screening. Several authors have suggested selective screening criteria for various settings. ${ }^{78}{ }^{29}$ However, suitable selection criteria could not been identified for asymptomatic infections among the general population in Amsterdam $^{30}$; thus, currently a comparison can only been made between a universal screening programme and no screening at all. 
The risk estimates for chlamydial infection, used in the decision model, differ from estimates used in previous cost effectiveness analyses. Previous studies have used estimates for PID and tubal factor infertility of $25-30 \%$ and $10-12 \%$, respectively.

However, no long term follow up studies of asymptomatic infections in a low prevalence population have yet been performed. Authors have based the estimates on studies among women with symptomatic infections, even though asymptomatic infections are most likely to be less virulent. In order to take that factor into account, lower estimates were used at baseline, and the estimates were varied over a wide range in the sensitivity analysis.

Similarly, the risk of a silent PID should be treated differently from the risk of an overt PID. Although several studies have attempted to study the association between silent PID and sequelae, ${ }^{31} 32$ there is not insufficient evidence to quantify any association. Consequently, it was not possible to make a distinction between silent and overt PID in the analysis. Instead, the baseline probability of developing sequelae after PID was adjusted, and a sensitivity analysis was performed.

The results of the sensitivity analysis underscore the importance of valid risk estimation, since this can have a substantial impact on the outcome of the analysis. In this study, the cost per MOA was extremely sensitive to variations in the assumption reflecting the chance of developing PID. Increasing the estimate from $5 \%$ to $25 \%$ resulted in a $78 \%$ decrease in cost per MOA. A similar effect was seen when the percentage of preventable PIDs was changed from $75 \%$ to $25 \%$. The model was also sensitive to the specificity of the test. No health gains or associated averted costs can be achieved among cases with a false positive test result; the costs decrease by $40 \%$ if the specificity of the test is estimated at $99.9 \%$ instead of $99 \%$. Variations in the estimates for ectopic pregnancy, tubal factor infertility, chronic pelvic pain, test sensitivity, and discount rate had much less impact.

COSTS CONSIDERED

All costs have been estimated for the Netherlands and converted into US\$. Cost in the model could reflect local differences in healthcare organisation and labour costs. Furthermore, the costs of health care and tests may change over time.

In the decision tree, the cost of all materials sent to participants and non-participants was included. Administrative costs, such as programme coordination, were also included. In practice, the costs will probably be higher, because of extra consultations for women who are worried about the potential consequences of the infection, the cost of media campaigns and other activities that would accompany the implementation of a programme. Furthermore, no physician fees for the screening were included, although other programmes-for example, influenza vaccination, that have been implemented in the Netherlands have included fees for physicians. ${ }^{33}$
In the analysis, complications of antibiotic treatment and referral and treatment of partners were not taken into consideration. Infected partners may reinfect females who have been screened, and can contribute to the spread of the infection in the population. It has been suggested recently, that men should always be included in a screening programme, and not just as "partners of infected women,"34 the reason for this being that targeting women only "stereotypes" them and affects their sexuality. Partner referral and screening aimed at men will be taken into account in a future evaluation of the effects of screening over time, using a more sophisticated dynamic model.

One hazard of cost effectiveness analysis is that it provides a unidimensional view of screening. The emphasis is placed on proving that the intervention is cost effective although, as this study shows, results can depend strongly on the assumptions included in a decision model. Moreover, other important aspects, such as invasion of privacy, psychological and social consequences of the test result, and the danger of medicalisation cannot be included in the model.

In conclusion, a universal screening programme for asymptomatic $C$ trachomatis infections among 15-40 year old women is not cost effective. Although the results of the analyses are sensitive to variation in the assumptions, the costs exceed the benefits even in the most optimistic scenario.

The authors would like to thank all practices and patients for taking the time to participate in the screening. This study was supported by the Zorg Onderzoek Nederland (previously called the Prevention Fund) grants 28-2588 and 28-1182-1.

Conflict of interest: none

1 Weström L, Joesoef R, Reynolds G, et al. Pelvic inflammatory disease and fertility; a cohort study of 1,844 women with laparoscopically verified disease and 657 control women with normal laparoscopic results. Sex Transm Dis 1992;4:185-91.

2 Westrom L. Incidence, prevalence and trends of acute pelvic inflammatory disease and its consequences in industrialized countries. Am F Obstet Gynecol 1980;138:880-91.

3 Schachter J, Grossman M, Sweet RL, et al. Prospective study of perinatal transmission of Chlamydia trachomatis. $\mathscr{F} A M A$ 1986;255:3374-7.

4 Scholes D, Stergachis A, Heidrich FE, et al. Prevention of pelvic inflammatory disease by screening for cervical chlamydial infection. N Engl f Med 1996;334:1362-6.

5 Kamwendo F, Forslin L, Bodin L, et al. Decreasing incidences of gonorrhea- and chlamydia-associated acute pelvic inflammatory disease. A 25-year study from an urban area of central Sweden. Sex Transm Dis 1996;23: 384-91.

6 Genç M, Ruusvaara L, Mardh PA. An economic evaluation of screening for Chlamydia trachomatis in adolescent males. $¥ A M A$ 1993;270:2057-64.

7 Marrazzo JM, Celum CL, Hillis SD, et al. Performance and cost-effectiveness of selective screening criteria for Chlamydia trachomatis infection in women. Implications for a national chlamydia control strategy. Sex Transm Dis for a national chlam $1997 ; 24: 131-41$.

8 Howell MR, Quinn TC, Gaydos CA. Screening for Chlamydia trachomatis in asymptomatic women attending family planning clinics. A cost-effectiveness analysis of three strategies. Ann Intern Med 1998;128:277-84.

9 Doornum GJ van, Buimer M, Prins M, et al. Detection of Chlamydia trachomatis infection in urine samples from men and women by ligase chain reaction. $\mathcal{F}$ Clin Microbiol 1995;33:2042-7.

10 Mouton JW, Verkooyen R, Meijden WI van der, et al. Detection of Chlamydia trachomatis in male and female urine specimens by using the amplified Chlamydia trachomatis test. F Clin Microbiol 1997;35:1369-72.

11 Østergaard L, Møller JK, Andersen B, et al. Diagnosis of urogenital Chlamydia trachomatis infection in women based on mailed samples obtained at home: multipractice comparative study. BMF 1996;313:1186-9.

12 Morré SA, Valkengoed IGM van, Jong A de, et al. Mailed, home-obtained urine specimens: a reliable screening home-obtained urine specimens: a reliable screening matis infections. f Clin Microbiol 1999;37:976-980. 
13 Nederlands Instituut voor Onderzoek van de Gezondheidszorg (NIVEL). Continuous Morbidity Registration Sentinel Stations the Netherlands 1997 (in Dutch). Utrecht: NIVEL, 1998.

14 Cates Jr W, Wasserheit JN. Genital Chlamydial infections: epidemiology and reproductive sequelae. Obstet Gynecol 1991;164:171-81

15 Centraal Bureau voor de Statistiek (CBS). Statistical yearbook of the Netherlands 1997. Voorburg: CBS, 1997.

16 Buhaug H, Skjeldestad FE, Halvorsen LS, et al. Should asymptomatic patients be tested for Chlamydia trachomatis in general practice? Br f Gen Pract 1990;40:142-5.

17 Postma MJ, Welte R, van den Hoek JA, et al. Opportunistic screening for genital infections with Chlamydia trachomatis in sexually active population of Amsterdam. II. Cost-effectiveness analysis of screening women [in Dutch] Ned Tijdschr Geneeskd 1999;143:677-81.

18 SIG Health Care Information Netherlands. Medical RegisIration, SIG, 1994

19 Centers for Disease Control and Prevention. Guidelines for treatment of sexually transmitted diseases. $M M W R$ 1998:47 (RR-1).

20 Severijnen AJ. Chlamydia trachomatis als volksgezondheidsprobleem (in Dutch). Rotterdam: Erasmus University, 1992.

21 Organisation for Economic Co-operations and Development. OECD Health Data for Windows, version 1997 (software). Paris: OECD, 1997

22 Hilgerman CN, Koopmanschap MA, Rutten FFH. Costs of intensive care in the Netherlands (in Dutch). Rotterdam: Erasmus University/iMTA, 1992.

23 Nederlands Ziekenhuis Insituut/Nederlandse Zorgfederatie. Inpatient Health Care in Figures as of fanuary 1st 1996 (in Dutch) Utrecht, Nederlands Ziekenhuis Instituut/ Nederlandse Zorgfederatie, 1996.
24 Ziekenfondsraad, Farmacotherapeutic Compass 1997 (in Dutch). Utrecht: Roto Smets, 1996.

25 Rutten FHH, Ineveld BM van, Ommen R van, et al. Cost estimation in health services research; guidelines for practice (in Dutch). Utrecht: Jan van Arkel, 1993.

26 Federal Statistical Office of Germany. Wages and salaries, survey about labour costs, series 16(5), salaries and wages in foreign countries 1996 (in German). Stuttgart: MetzlerPoeschel, 1997.

27 Gold MR, Siegel JE, Russel LB, et al. Cost-effectiveness analysis in health and medicine. Oxford: Oxford University Press, 1997.

28 Parsonage M, Neuberger H. Discounting and health benefits. Health Econ 1992;1:71-6.

29 Grun L, Tassano-Smith J, Carder C, et al. Comparison of two methods of screening for genital chlamydial infection in women attending in general practice:cross sectional survey. $B M F$ 1997;315:226-30.

30 Valkengoed IGM van, Morré SA, Brule AJC van den, et al. Low diagnostic accuracy of selective screening criteria for asymptomatic Chlamydia trachomatis infections in the general population. Sex Transm Inf 2000;76:375-380.

31 Sellors JW, Mahony JB, Chernesky MA, et al. Tubal factor infertility:an association with prior chlamydial infection infertility:an association with prior chlamydial infection

32 Brunham RC, Binns B, McDowell J, et al. Chlamydia trachomatis infection in women with ectopic pregnancy.
Obstet Gynecol 1986;67:722-6

33 Postma MJ, Bor JM, Gennep M van, et al. Economic evaluation of influenza vaccination; assessment for the Netherlands. PharmacoEconomics 1999;16(Suppl 1):33-40.

34 Duncan B, Hart G. Sexuality and health:the hidden costs of screening for Chlamydia trachomatis. BMf 1999;318:9313.

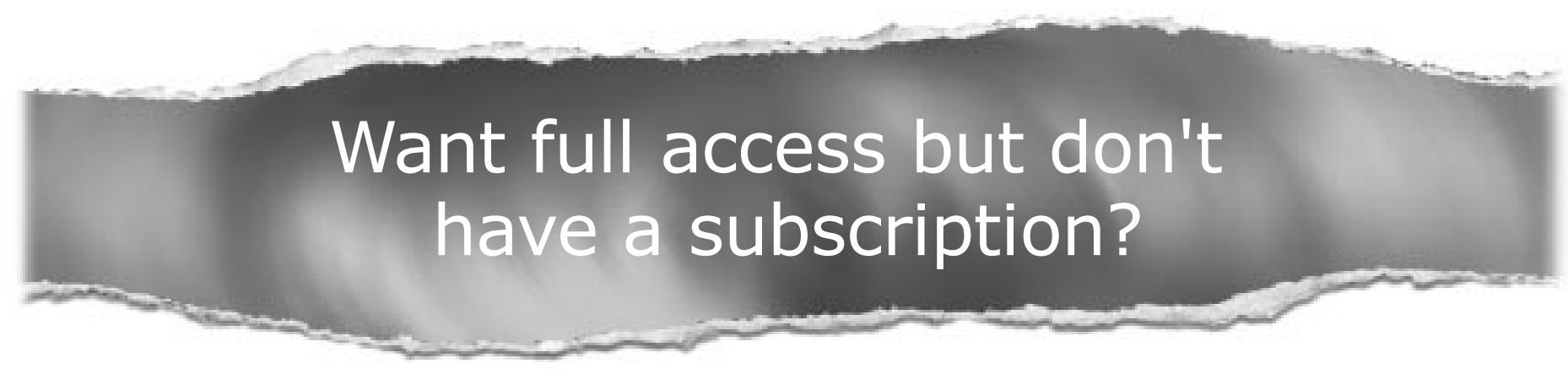

\section{Pay per access}

For just US $\$ 25$ you can have instant access to the whole website for 30 days. During this time you will be able to access the full text for all issues (including supplements) available. You will also be able to download and print any relevant pdf files for personal use, and take advantage of all the special features Sexually Transmitted Infections online has to offer.

\section{www.sextransinf.com}

